\title{
Déterminants des itinéraires de transhumance à la périphérie de la réserve de biosphère transfrontalière du W au Bénin
}

\author{
Byll O. KPEROU GADO*1\&4, Ismaïla TOKO IMOROU2, Ousséni AROUNA³, Habirou SIDI IMOROU1 et \\ Madjidou OUMOROU4 \\ ${ }^{1}$ Ministère de l'Agriculture, de l'Elevage et de la Pêche, 03 BP : 2900 Cotonou, Bénin. \\ 2 Laboratoire de Cartographie "LaCarto"/Université d'Abomey-Calavi, 10 BP 1082 Cotonou-Houéyiho, Bénin. \\ 3 Ecole Nationale Supérieure des Travaux Publics (ENSTP), Université Nationale des Sciences, Technologies, \\ Ingénierie et Mathématiques d'Abomey (UNSTIM), BP : 2282, Goho Abomey, Bénin. \\ 4 Laboratoire de Recherche en Biologie Appliquée (LARBA), Unité de Recherche en Phytosociologie, Espaces \\ Protégés et Pastoraux, Agro-écosystèmes, Conservation et valorisation des espèces endogènes (PEPPAC), Ecole \\ Polytechnique d'Abomey-Calavi, BP 2009 Cotonou, Bénin. \\ *Auteur correspondant - Email : kperoubyll2014@gmail.com
}

Original submitted in on $1^{\text {th }}$ June 2020. Published online at www.m.elewa.org/journals/ on $31^{\text {st }}$ August 2020 https://doi.org/10.35759/JABs.152.5

\section{RESUME}

Objectifs : la présente étude vise à identifier les facteurs concourant à la définition des itinéraires de transhumance et analyser la perception des éleveurs transhumants sur les déterminants de ces itinéraires en vue d'une amélioration de la prise de décision dans la gestion des écosystèmes pâturés.

Méthodologie et résultats : la démarche méthodologique a consisté à cartographier, avec le logiciel ArcGIS 10.4, les infrastructures pastorales et à faire une enquête socioéconomique de la perception des transhumants sur la situation et la gestion des couloirs. Les résultats indiquent l'existence de couloirs locaux ou communaux, intercommunaux et régionaux autour de la réserve. II existe des liens significatifs entre couloirs et points d'eau, postes vétérinaires frontaliers, marchés à bétail, parcs de vaccination, pharmacies et cabinets vétérinaires. Dans certaines zones, les couloirs n'existent pas, sont obstrués, non fonctionnels ou confondus aux pâturages. Les itinéraires sont dynamiques et régressent numériquement avec le temps du fait de la dégradation des parcours, de l'obstruction des couloirs, des conflits et de l'assèchement des points d'eau. L'histoire, la culture et les institutions aussi sont déterminants.

Conclusions et application des résultats : les déterminants des itinéraires de transhumance à la périphérie de la réserve du W sont historiques, culturels, environnementaux, socio-économiques et institutionnels. Les différents types de couloirs sont interconnectés. Une gestion rationnelle, apaisée et durable des itinéraires de transhumance nécessite une connaissance approfondie de ces déterminants dans les écosystèmescibles. La mise en place d'une base de données permettrait aux autorités à divers niveaux d'avoir une vue d'ensemble sur la répartition de ces infrastructures et des outils d'aide à la décision pour une meilleure valorisation du pastoralisme et un meilleur suivi des ressources et infrastructures pastorales.

Mots clés : couloirs de transhumance, représentation cartographique, analyse de perception, réserve de biosphère transfrontalière du W du Bénin. 


\title{
Determinants of transhumance corridors at the periphery of the transboundary biosphere reserve of
} W in Benin

\begin{abstract}
Objectives: this study aims to identify the factors contributing to the definition of transhumance corridors and analyse the perception of transhumant herders on the determinants of these corridors with a view to improving decision-making in the management of grazed ecosystems.

Methodology and results: the methodological approach consisted on mapping, with ArcGIS 10.4 software, pastoral infrastructures and carrying out a socio-economic survey of the perception of transhumants on the situation and management of the corridors. The results indicate the existence of local or municipal, intermunicipal and regional corridors around the reserve. There are significant links between corridors and water points, border veterinary posts, livestock markets, vaccination parks, pharmacies and veterinary surgeries. In some areas, the corridors do not exist, are obstructed, non-functional or confused with pastures. The corridors are dynamic and regress numerically over time due to the deterioration of the pastures, the obstruction of the corridors, conflicts and the drying up of water points. History, culture and institutions are also crucial.

Conclusions and application of results: the determinants of the transhumance corridors on the periphery of the $\mathrm{W}$ reserve are historical, cultural, environmental, socio-economic and institutional. The different types of corridors are interconnected. Rational, peaceful and sustainable management of transhumance routes requires an in-depth knowledge of these determinants in the target ecosystems. The establishment of a database would allow authorities at various levels to have an overview of the distribution of these infrastructures and decision-making tools for better valorisation of pastoralism and better monitoring of pastoral resources and infrastructures.
\end{abstract}

Keywords: transhumance corridors, cartographic representation, perception analysis, Benin W transboundary biosphere reserve.

\section{INTRODUCTION}

L'utilisation extensive des parcours communautaires pour l'élevage représente en Afrique subsaharienne, un mode de vie culturel et économique de 10 à $25 \%$ de la population totale des régions concernées en Afrique et permet à des millions d'exploitants familiaux de vivre dignement dans des contextes difficiles (Nori et al., 2008; Duteurtre et Faye, 2009). La contribution de l'élevage au PIB national au Bénin est de $6 \%$, soit $16 \%$ du PIB agricole qui lui, représente $32 \%$ du PIB national en 2011 (Codjia, 2016). Cet élevage concerne surtout les bovins, les ovins, les caprins et les volailles. Les projections entre 2015 et 2019 montrent que le cheptel ruminant est passé de 662537 à 734175 têtes de bovins et de 358280 à 390864 têtes de petits ruminants (DPA, 2016). Malgré cette importance, le système d'élevage demeure traditionnel, extensif, avec une productivité tributaire de la disponibilité en pâturage et en eau qui commande les mouvements de

troupeaux et définit ce mode de production transhumant (Eboh et al., 2008). Cette transhumance est actuellement confrontée à de graves contraintes, notamment, l'incertitude climatique, les pressions liées à la croissance démographique, la concurrence en saison sèche des animaux transhumants venants des pays limitrophes (Niger, Nigeria, Burkina Faso) et les politiques publiques qui précarisent l'accès aux ressources pastorales et compromettent la mobilité du bétail (Djènontin et al., 2009 ; Katè et al., 2014 ; Zakari et al., 2015). Face à la diminution de la biomasse fourragère et à la baisse progressive de la valeur nutritive des pâturages en saison sèche, les stratégies pour l'utilisation des parcours naturels intègrent plusieurs facteurs dont la modification des itinéraires (Kagone et al., 2006; Kpérou Gado, 2006 ; Lesse et al., 2015). Cette étude se propose d'identifier les facteurs concourant à la définition des itinéraires de transhumance et d'analyser la 
perception des éleveurs sur ces itinéraires en vue de propositions pour une amélioration de la gestion des parcours à la périphérie de la réserve de

\section{MATERIEL ET METHODES}

Milieu d'étude : Les données ont été collectées à la périphérie de la RBTW-B dans l'extrême nord du Bénin, entre $10^{\circ} 30^{\prime}$ et $12^{\circ} 24^{\prime}$ de latitude nord et $01^{\circ} 39^{\prime}$ et $03^{\circ} 36^{\prime}$ de longitude est (figure 1). Cette périphérie couvre les communes de Karimama, Malanville, Kandi, Banikoara et Kérou sur une superficie de $13042,62 \mathrm{~km}^{2}$. Elle est représentée par la zone tampon de la RBTW-B (zone à usage contrôlé) et la zone libre. Le secteur d'étude situé dans la région soudanienne, fait partie des districts phytogéographiques de la Mékrou-Pendjari, de la Chaîne de l'Atacora et du Borgou-Nord (Adomou et al., 2006). La périphérie de la RBTW-B est sous l'influence d'un climat tropical de type soudanien caractérisé par deux grandes saisons, une saison sèche biosphère transfrontalière du $W$ du Bénin (RBTW$B$ ). Elle se veut être un outil d'aide à la décision pour améliorer la gestion des écosystèmes pâturés.

d'octobre à avril et une saison des pluies de mai à septembre. La pluviosité moyenne annuelle oscille entre $677 \mathrm{~mm}$ au nord et $1060 \mathrm{~mm}$ au sud; les températures moyennes mensuelles sont comprises entre $25^{\circ} \mathrm{C}$ au sud et $33^{\circ} \mathrm{C}$ au nord (Météo-Bénin, 2018). Le réseau hydrographique est assez diversifié et comprend des cours d'eau dont les plus importants sont la Mékrou et l'Alibori qui se jettent dans le fleuve Niger. La végétation de la RBTW-B et sa périphérie est essentiellement constituée de forêts galeries, de forêts claires, de savanes boisées, arborées, arbustives et herbeuses, de champs, de jachères et de plantations forestières (Houessou et al., 2013).

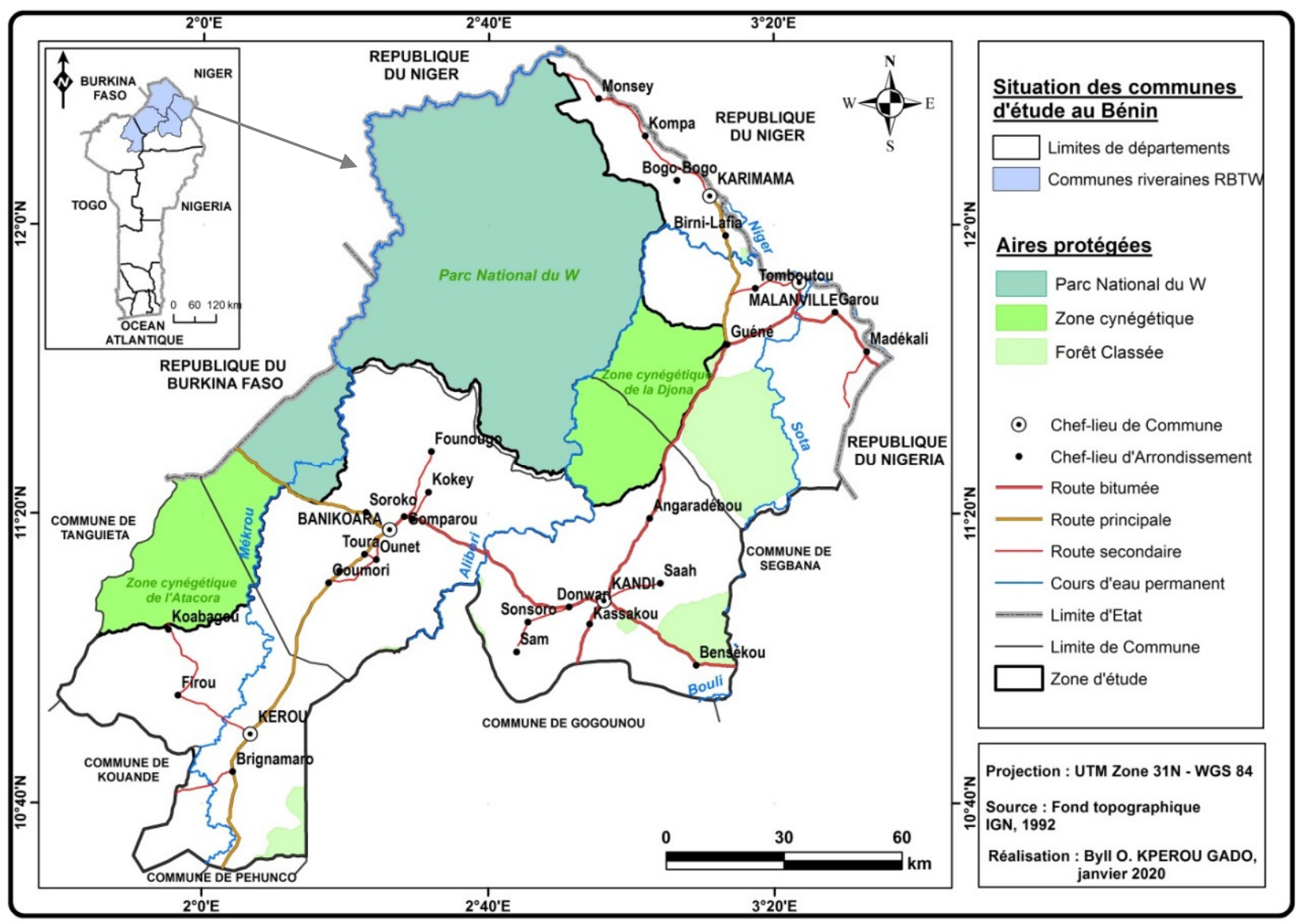

Figure 1 : Zone d'étude 


\section{Méthodes}

Cartographie des itinéraires de transhumance et autres infrastructures pastorales : Les coordonnées géographiques des couloirs de transhumance, des ressources et infrastructures pastorales (pâturages, points d'eau, marchés à bétail, postes vétérinaires frontaliers, parcs de vaccination, pharmacies et cabinets vétérinaires) sont tirées de la littérature ou enregistrées sur le terrain à l'aide d'un récepteur GPS (Global Positioning System) de marque GARMIN ${ }^{\circledR}$ etrex VISTA $\mathrm{HCx}$, de précision 5 mètres. Elles ont été exploitées pour réaliser, à l'aide du logiciel ArcGIS 10.4, des cartes des principaux axes de transhumance, des ressources exploitées et des infrastructures d'appui à cette pratique pastorale à la périphérie de la RBTW-B.

Identification des déterminants des itinéraires de transhumance et analyse de la perception des itinéraires : Pour comprendre et évaluer la dynamique spatio-temporelle des itinéraires de transhumance dans les parcours de la zone d'étude et analyser la perception des déterminants de ces mutations à la périphérie de la RBTW-B, les informations sur les couloirs de transhumance (périodes d'aménagement, historique des positions, fonctionnalité, circonstances des changements) ont été collectées à l'aide d'un questionnaire d'enquête adressé aux éleveurs transhumants. Pour une triangulation des informations recueillies, des personnes ressources qui détiennent des informations fiables sur ces itinéraires de transhumance dans la zone d'étude (autorités locales, services techniques déconcentrés de l'Etat, membres des comités de gestion de la transhumance) ont été interviewées sur la base de guides d'entretien. Le choix des unités primaires d'enquêtes (hameaux, campements, couloirs, aires de pâturages, points d'eau, marchés à bétail, lieux de vaccination) est raisonné et basé sur l'accessibilité. Les unités secondaires que sont les éleveurs transhumants sont retenues de façon aléatoire. La taille de l'échantillon enquêté au niveau de chaque commune a été déterminée par la formule suivante : $\mathbf{n}=\frac{\mathbf{U}^{2}{ }_{\mathbf{1 - \alpha} / \mathbf{2}} \times \mathbf{p}(\mathbf{1}-\mathbf{p})}{\mathbf{d}^{\mathbf{2}}}$ (Dagnelie, 2007) ; n est la taille de l'échantillon; $U_{1-\alpha / 2}$ est la valeur de la variable aléatoire normale pour une valeur de la probabilité $(1-\alpha / 2)=0,975 ; U^{2}{ }_{1-\alpha / 2}=(1,96)^{2}=3,84 ; p$ est la proportion de chaque catégorie d'acteurs obtenue à partir d'une enquête exploratoire; $d=10 \%$ est la marge d'erreur fixée en tenant compte de la précision voulue. La proportion des catégories d'acteurs par commune riveraine suite à l'enquête exploratoire et la répartition des différents acteurs rencontrés à la phase des enquêtes approfondies sont détaillées dans le Tableau 1.

Tableau 1 : Proportions et taille de l'échantillon des éleveurs transhumants enquêtés par commune

\begin{tabular}{l|c|c|c|c|c|c}
\hline \multirow{2}{*}{ PARAMETRES } & \multicolumn{5}{c|}{ COMMUNES } & \multirow{2}{*}{ TOTAL } \\
\cline { 2 - 7 } & Kérou & Banikoara & Kandi & Malanville & Karimama & \\
\hline Proportions (\%) & 24 & 20 & 18 & 15 & 23 & 100 \\
Taille de l'échantillon & 55 & 45 & 40 & 33 & 52 & 225 \\
\hline
\end{tabular}

Les données d'enquêtes ont été analysées à l'aide du logiciel Statistical Package for Social Sciences (SPSS), version 21 et sous XLSTAT, version 2014.5.03. Des analyses descriptives (taux moyen de réponse, moyennes, écarts-types, graphiques), exploratoires (analyse factorielle des correspondances) et des tests

\section{RESULTATS \\ Cartographie des itinéraires de transhumance et autres infrastructures pastorales}

Réseaux de couloirs de transhumance: Les itinéraires de transhumance identifiés dans la zone riveraine de la RBTW-B permettent de distinguer les couloirs de transhumance en fonction de leur envergure territoriale en couloirs locaux, communaux, intercommunaux et régionaux (Tableau 2). Les couloirs locaux sont dans les limites des terroirs villageois et statistiques ont été faits, notamment des tests de corrélation entre variables. Ces tests ont permis de déterminer les variables qui étaient significativement corrélées entre elles $(p \leq 0,5)$ et de choisir les plus pertinentes en vue d'identifier les déterminants des itinéraires de transhumance.

permettent aux éleveurs de quitter leur campement pour la petite transhumance. Les couloirs communaux ou intercommunaux peuvent raccorder respectivement plusieurs arrondissements d'une commune ou plusieurs communes; ils sont empruntés pour la petite ou la grande transhumance. Enfin, les couloirs régionaux sont ceux qui permettent la circulation des animaux d'un pays à l'autre dans la sous-région ouest-africaine pour la grande transhumance. Sur $1524 \mathrm{~km}$ de couloirs 
répertoriés autour de la RBTW-B, les couloirs locaux ou communaux représentent $33 \%$, ceux intercommunaux
$24 \%$ et les couloirs régionaux $43 \%$. Ils sont interconnectés (Figure 2).

Tableau 2 : Couloirs de transhumance classés par types et selon les itinéraires

\begin{tabular}{|c|c|c|c|c|}
\hline TYPES & $\begin{array}{l}\text { PAYS/COMMUNES } \\
\text { TRAVERSES }\end{array}$ & ITINERAIRES & IDENTIFIANTS & $\begin{array}{l}\text { LONGUEUR } \\
(\mathrm{km})\end{array}$ \\
\hline Régionaux & $\begin{array}{l}\text { Burkina-Banikoara-Kérou- } \\
\text { Sinendé } \\
\text { Burkina-Banikoara-Kandi- } \\
\text { Gogounou } \\
\text { Niger-Karimama-Malanville } \\
\text { Niger-Karimama-Malanville } \\
\text { Niger-Karimama-Malanville- } \\
\text { Ségbana } \\
\text { Niger-Malanville } \\
\text { Niger-Malanville } \\
\text { Nigéria-Malanville-Ségbana }\end{array}$ & $\begin{array}{l}\text { Axe } 3 \text { CEDEAO } \\
\text { Burkina-Mékrou-Petit Paris } \\
\text { Axe } 4 \text { CEDEAO } \\
\text { Niger-Kargui-Kangara peulh } \\
\\
\text { Niger-Mékrou-FC Sota } \\
\text { Axe } 5 \text { CEDEAO } \\
\text { Niger-Kambouwotounga- } \\
\text { Karamba } \\
\text { Nigéria-FC Goungoun et Sota }\end{array}$ & $\begin{array}{l}\text { Cedeao_3 } \\
\text { Reg_Bf_gn } \\
\text { Cedeao_4 } \\
\text { Reg_Nr_ml } \\
\text { Reg_Nr_sg } \\
\text { Cedeao_5 } \\
\text { Reg_Nr_ml } \\
\text { Reg_Ng_sg }\end{array}$ & $\begin{array}{l}123 \\
77 \\
131 \\
16 \\
168 \\
54 \\
\\
56 \\
28\end{array}$ \\
\hline $\begin{array}{l}\text { Intercom- } \\
\text { munaux }\end{array}$ & $\begin{array}{l}\text { Banikoara-Kérou-Kouandé- } \\
\text { Djougou } \\
\text { Banikoara-Gogounou } \\
\text { Kandi-Gogounou } \\
\text { Banikoara-Gogounou } \\
\text { Banikoara-Gogounou } \\
\text { Banikoara-Kérou } \\
\text { Kérou-Kouandé } \\
\text { Kérou-Kouandé }\end{array}$ & $\begin{array}{l}\text { Guimbagou-FC Mékrou } \\
\text { Bontè-Petit Paris } \\
\text { Bodérou-Gounarou } \\
\text { Goumori-Alibori } \\
\text { Gbassabansou-Alibori } \\
\text { Founougo-Amanki } \\
\text { Firou-Nassoukou } \\
\text { Amanki-Ouroufina }\end{array}$ & $\begin{array}{l}\text { Int_bk_dj } \\
\text { Int_bk_gn } \\
\text { Int_kd_gn } \\
\text { Int_bk_gn1 } \\
\text { Int_bk_gn2 } \\
\text { Int_bk_kr } \\
\text { Int_kr_kn1 } \\
\text { Int_kr_kn2 }\end{array}$ & \begin{tabular}{|l}
114 \\
47 \\
9 \\
25 \\
12 \\
107 \\
13 \\
45 \\
\end{tabular} \\
\hline \multirow{4}{*}{$\begin{array}{l}\text { Locaux } \\
\text { ou com- } \\
\text { munaux }\end{array}$} & $\begin{array}{l}\text { Kérou } \\
\text { Banikoara }\end{array}$ & \begin{tabular}{|l|} 
Djoléni-Gorobani \\
Founougo-Kigarou \\
Soroko-Mondoukoka \\
Founougo-Gomparou
\end{tabular} & \begin{tabular}{|l|} 
Loc_kr1 \\
Loc_bk1 \\
Loc_bk2 \\
Loc_bk3
\end{tabular} & \begin{tabular}{|l|}
7 \\
39 \\
20 \\
22
\end{tabular} \\
\hline & Kandi & $\begin{array}{l}\text { Donwari-Sam } \\
\text { Alfakoara-Bensékou } \\
\text { Kofoïssa-Bénin peulh } \\
\text { Mongo-Kourobourowinrayérou } \\
\text { Gambanè-Kassakou- } \\
\text { Oroutoura } \\
\text { Dodoma-Dowari }\end{array}$ & $\begin{array}{l}\text { Loc_kd1 } \\
\text { Loc_kd2 } \\
\text { Loc_kd3 } \\
\text { Loc_kd4 } \\
\text { Loc_kd5 } \\
\text { Loc_kd6 }\end{array}$ & $\begin{array}{l}22 \\
60 \\
39 \\
11 \\
\\
23 \\
14\end{array}$ \\
\hline & Malanville & $\begin{array}{l}\text { Zone Guéné } \\
\text { Damazotounga-Soumbégorou } \\
\text { peulh } \\
\text { Fiafounfoun-Massangari } \\
\text { Bangou-Goungoun } \\
\text { Kangara peulh-Molla } \\
\text { Malanville-Dèguèdèguè } \\
\text { Sakawan zénon-Wollo }\end{array}$ & $\begin{array}{l}\text { Loc_ml1 } \\
\text { Loc_ml2 } \\
\text { Loc_ml3 } \\
\text { Loc_ml4 } \\
\text { Loc_ml5 } \\
\text { Loc_ml6 } \\
\text { Loc_ml7 }\end{array}$ & $\begin{array}{l}45 \\
30 \\
29 \\
16 \\
10 \\
7 \\
7 \\
\end{array}$ \\
\hline & Karimama & $\begin{array}{l}\text { Farantounga-Tchakorga } \\
\text { Mamassy peulh-Kara } \\
\text { Tondigamè-Mékrou } \\
\text { Pétchinga-Loumbouloumbou } \\
\text { Koudjibangou-Barou }\end{array}$ & $\begin{array}{l}\text { Loc_km1 } \\
\text { Loc_km2 } \\
\text { Loc_km3 } \\
\text { Loc_km4 } \\
\text { Loc_km5 }\end{array}$ & \begin{tabular}{|l|}
12 \\
18 \\
3 \\
18 \\
13
\end{tabular} \\
\hline
\end{tabular}




\begin{tabular}{l|l|l|l|l}
\hline \multirow{2}{*}{ TYPES } & $\begin{array}{l}\text { PAYS/COMMUNES } \\
\text { TRAVERSES }\end{array}$ & ITINERAIRES & IDENTIFIANTS & $\begin{array}{l}\text { LONGUEUR } \\
(\mathbf{k m})\end{array}$ \\
\hline & & Tchantagaré-Kombayigata & Loc_km6 & 11 \\
& & Bogobogo-Toura & Loc_km7 & 8 \\
& & Kara-Kompanti-FI Niger & Loc_km8 & 7 \\
& Goroukambou-Tondikoaria & Loc_km9 & 11 \\
\hline
\end{tabular}

Points d'eau naturels et artificiels à but pastoral à la périphérie de la RBTW-B : La figure 2 présente la répartition des points d'eau naturels et artificiels à but pastoral à la périphérie de la RBTW-B. Cette figure indique en outre, les liens entre ces ressources hydriques, les couloirs de transhumance et les aires de pâturage.

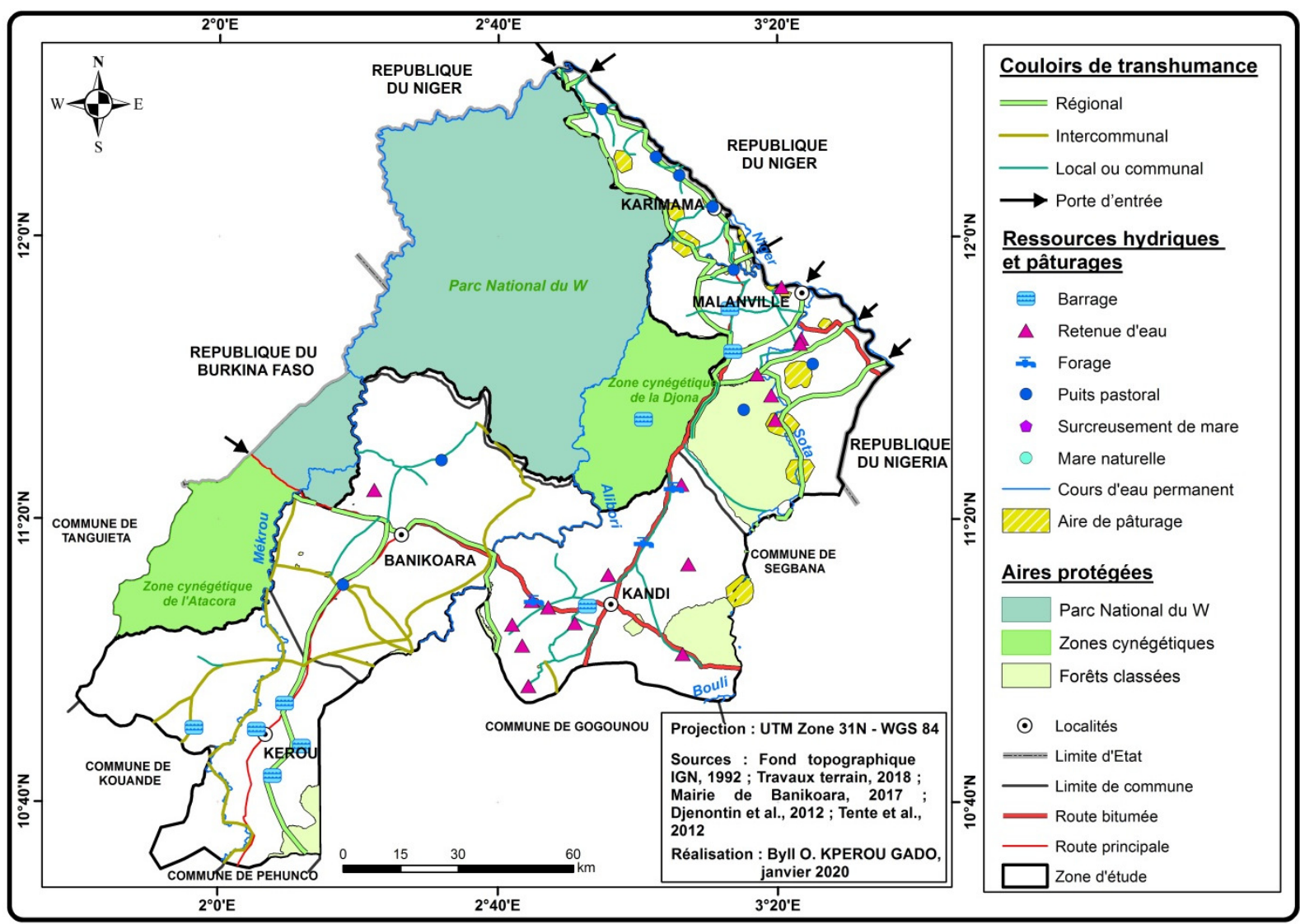

Figure 2 : Itinéraires de transhumance, ressources hydriques à but pastoral et pâturages à la périphérie de la réserve de biosphère transfrontalière du $\mathrm{W}$

Les cours d'eau sont principalement le fleuve Niger et ses affluents (Mékrou, Alibori, Sota et Bouli) qui sont les cours d'eau permanents à la périphérie de la réserve. La trajectoire de ces affluents impacte significativement les itinéraires des animaux pour la grande transhumance dans la zone d'étude. Quant aux cours d'eau temporaires de la zone, ils sont essentiellement fréquentés au cours de la petite transhumance et très peu exploités par les animaux au moment de la grande transhumance en raison de leur assèchement. L'accès au cours d'eau à l'intérieur de la réserve est interdit et réprimé. Les points d'eau artificiels à but pastoral dans la zone d'étude sont des barrages, retenus d'eau, forages, puits pastoraux, surcreusements de mares ou des mares naturelles. Ces sont des infrastructures hydrauliques réalisées au profit du bétail, de la pêche ou des besoins agricoles. Ces points d'eau artificiels sont diversement répartis. Dans la commune de Kérou, il y a 
principalement des barrages. Dans la commune de Banikoara, la majorité des infrastructures d'hydraulique pastorale sont des surcreusements de mares, alors que dans celle de Kandi, il s'agit plus de retenues d'eau. La commune de Malanville a une diversité d'infrastructures d'hydraulique pastorale, tandis que dans Karimama, il n'y a que des puits pastoraux. Globalement, quatre principaux types de points d'eau pastoraux sont exploités par les animaux transhumants à la périphérie de la RBTW-B. II s'agit des cours d'eau naturels (selon
$92,9 \%$ des éleveurs transhumants), des forages ou puits pastoraux $(50,2 \%)$, des barrages $(42,2 \%)$ et des surcreusements de mares (16,4\%). La figure 2 montre que plusieurs points d'eau sont sur les axes de transhumance; mais, ils sont en nombre insuffisant dans la zone d'étude, selon $85,6 \%$ des transhumants; $92,1 \%$ des éleveurs estiment qu'ils sont en outre éloignés de leurs aires de parcours. La figure 3 représente les types de points d'eau exploités autour de la RBTW-B.

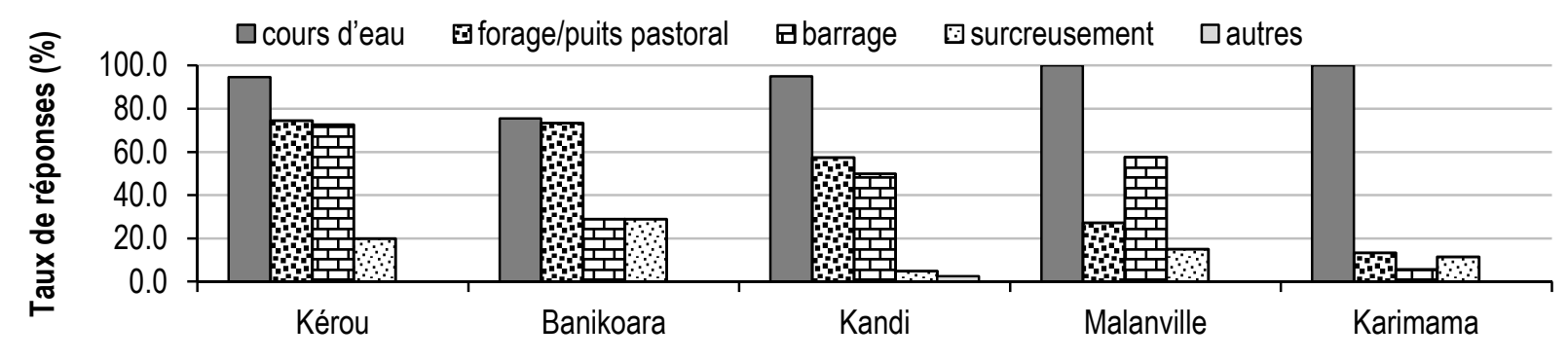

Communes périphérique de la RBTW-B

Figure 3 : Types de points d'eau exploités dans les communes périphériques de la RBTW-B

Elle indique que $100 \%$ des transhumants dans la zone de Malanville et Karimama exploitent des points d'eau naturels. L'accès aux autres sources d'abreuvement est très limité à Karimama, alors que dans les autres communes, les sources d'abreuvement sont plus diversifiées, avec quelques différences. Ces différences sont confirmées par un test de $\mathrm{khi}^{2}$ qui montre l'existence, au seuil de $5 \%$, d'une association très significative entre les communes traversées par les transhumants et les types de points d'abreuvement exploités par leurs animaux $\left(\mathrm{khi}^{2}=183,9 ; \mathrm{ddl}=32 ; \mathrm{p}<\right.$ 0,001 et $V$ de Cramer $=0,45$ ).

Postes vétérinaires frontaliers, marchés à bétail, parcs de vaccination et pharmacies/cabinets vétérinaires : La figure 4 représente les infrastructures pastorales autour de la RBTW-B. Ces infrastructures ont des liens avec les axes de transhumance. En effet, les animaux transhumants ont l'obligation de rentrer sur le territoire national par un poste vétérinaire frontalier. Dans la zone d'étude, les postes officiels de contrôle sont Madécali, Malanville, Monsey et Mékrou. La transhumance, notamment celle commerciale tient grand compte de la situation des marchés à bétail dans le choix des itinéraires. Presque tous les marchés à bétail de la zone d'étude (17 recensés) sont sur les grands axes de transhumance. La santé des animaux oblige les transhumants à recourir aux unités sanitaires vétérinaires. Les positions géographiques de ces unités sont influencées par les itinéraires de transhumance et la position des agglomérations. Les éleveurs se déplacent vers ces unités pour les approvisionnements ou sollicitent les services des cliniques vétérinaires ambulantes. Dans la zone d'étude, 15 centres, cabinets et pharmacies vétérinaires privés ou publics sont recensés. Les structures vétérinaires des zones traversées imposent le recours aux parcs ou aires de vaccination quand les animaux en transhumance n'ont pas fait certaines vaccinations obligatoires avant leur départ des zones d'attache. Les parcs de vaccination sont érigés en matériaux définitifs dans les communes de Karimama, Malanville et dans une moindre mesure, dans la commune de Kandi. Ils sont situés à MamassyPeulh, Kargui, Kogoungou, Illa, Garbey-Koara, Kompanti, Monsey, Pétchinga, Guéné, Férébangou, Boilfo, Kassa et Alfakoara. Dans les autres communes, il y a plutôt des aires de regroupement retenues de commun accord avec les services vétérinaires et éleveurs pour les campagnes de vaccination. Plus de 160 aires sont recensées dans la commune de Banikoara. 


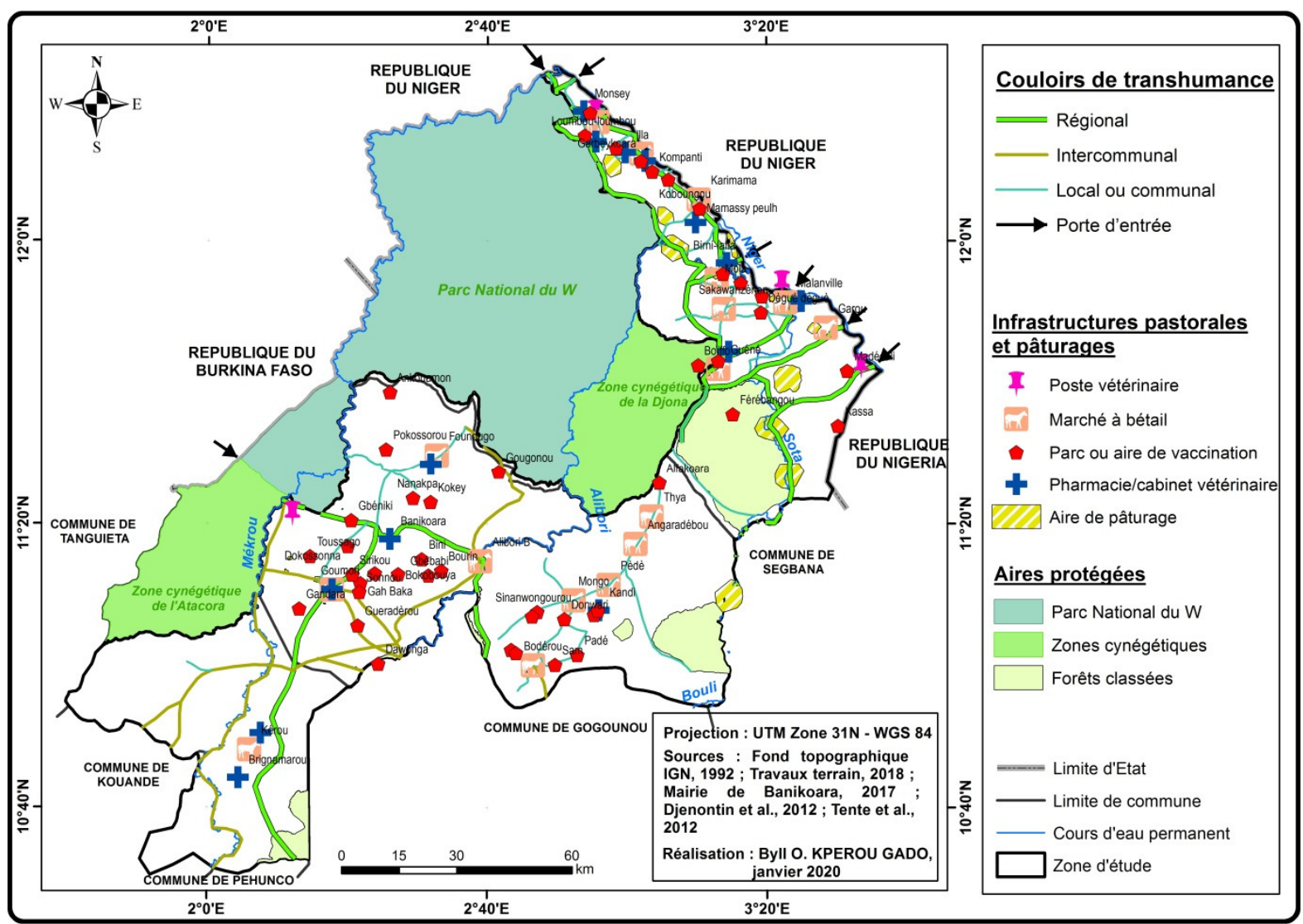

Figure 4 : Itinéraires de transhumance, infrastructures pastorales et pâturages à la périphérie de la réserve de biosphère transfrontalière du $\mathrm{W}$

Perception des éleveurs sur la situation des couloirs de transhumance autour de la RBTW-B

Description des couloirs de transhumance par les éleveurs dans la zone d'étude : Les éleveurs transhumants autour de la RBTW-B connaissent les itinéraires de transhumance et ont plusieurs approches pour les décrire. Ces itinéraires sont clairement identifiés à travers des repères ponctuels (localités), linéaires (routes) ou des espaces (aires protégées) par 37,4\% des transhumants ; $29,8 \%$ les décrivent en se référant aux structures réalisatrices (structures officielles locales, projets/programmes d'élevage, Communauté Economique des Etats de l'Afrique de l'Ouest notamment) ; $25,7 \%$ des éleveurs enquêtés estiment que les couloirs n'existent pas parce que obstrués, non fonctionnels ou confondus aux aires de pâture ; enfin, pour $7,0 \%$, il s'agit de couloirs naturels, non balisés (figure $5 \mathrm{a}$ ). 


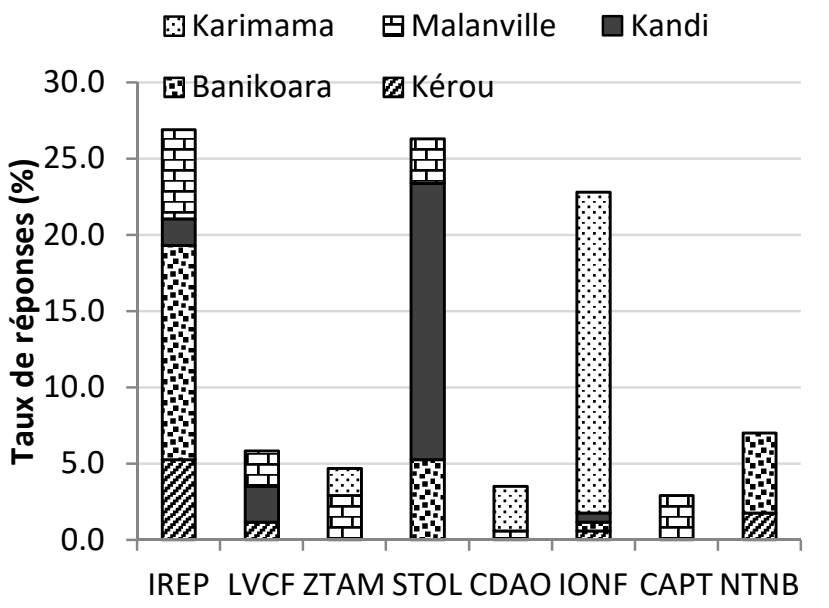

Types de couloirs

\section{(a) Diagramme en bâtons superposés}

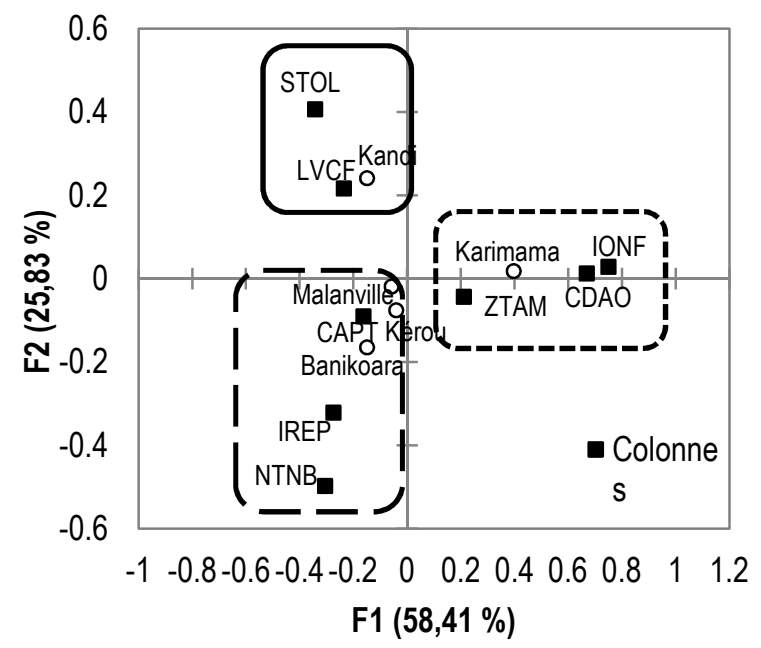

(b) Graphique symétrique (axes F1 et F2 : 84,24 \%)

Figure 5 : Description des couloirs de transhumance dans la zone d'étude

Légende - IREP : couloirs clairement identifiés par des repères ; STOL : couloirs tracés par des structures officielles locales ; CDAO : couloirs CEDEAO ; LVCF : couloirs le long des voies, champs ou forêts ; NTNB : couloirs naturels, non balisés ; ZTAM : couloirs conduisant à la zone tampon ; IONF : couloirs inexistants, obstrués ou non fonctionnels ; CAPT : couloirs confondus aux aires de parcours.

Une analyse factorielle des correspondances (AFC) montre qu'il existe un lien très significatif entre les types de couloirs décrits et les communes riveraines de la RBTW-B traversées par les éleveurs transhumants $\left(\mathrm{khi}^{2}\right.$ $=269,65 ; \mathrm{ddl}=28 ; \mathrm{p}$-value $<0,0001)$. Il se dégage trois grands regroupements par rapport à la perception des éleveurs sur les types de couloirs empruntés (figure $5 b$ ) ; il s'agit des regroupements

(i) Kérou, Banikoara et Malanville où les couloirs sont confondus aux aires de parcours en majorité ; mais ces couloirs sont soit clairement identifiés par des repères ou sont des couloirs naturels, non balisés ;

(ii) Kandi où les couloirs sont décrits comme des espaces situés en bordure des champs, forêts ou des routes (bitumées, non bitumées ou pistes); certains de ces couloirs sont validés et aménagés par les structures officielles locales et ;

(iii) Karimama où les éleveurs utilisent des couloirs officiels tels que celui de la CEDEAO et des couloirs conduisant à la Zone tampon de la RBTW-B ; ces couloirs sont toutefois obstrués par des occupations anarchiques d'habitation et d'exploitations agricoles. Dynamique temporelle de l'identification et de la matérialisation des couloirs de transhumance : Les informations sur l'évolution dans le temps de l'identification et de la matérialisation des couloirs dans la zone de la RBTW-B sont connues de $60 \%$ des éleveurs transhumants rencontrés (figure 6a). D'après ces informations, les premières matérialisations de couloirs de transhumance datent d'avant les années 1970 ( $1 \%$ des avis). Ces réalisations ont évolué graduellement dans le temps et se sont intensifiées les vingt dernières années (2000-2018 pour 48\% des avis). Les communes de Banikoara et Malanville sont vraisemblablement les pionnières dans ces initiatives ; la commune de Kandi a connu la grande partie des réalisations les dix dernières années (2010-2018 pour $15 \%$ des avis); quant à Kérou et Karimama, peu d'informations sont connues sur l'évolution temporelle de l'identification et de la matérialisation des couloirs. 


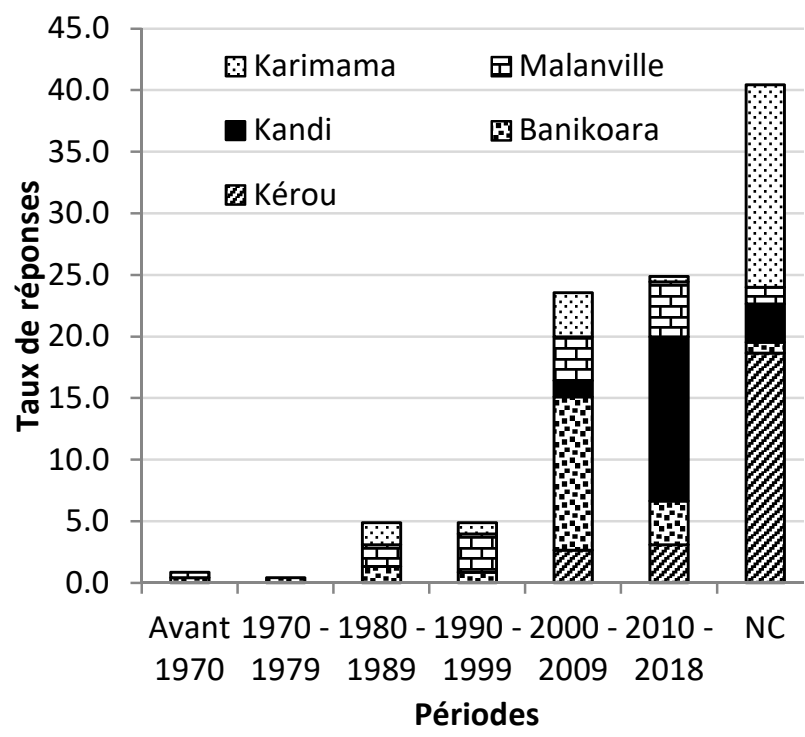

(a) Diagramme en bâtons superposés

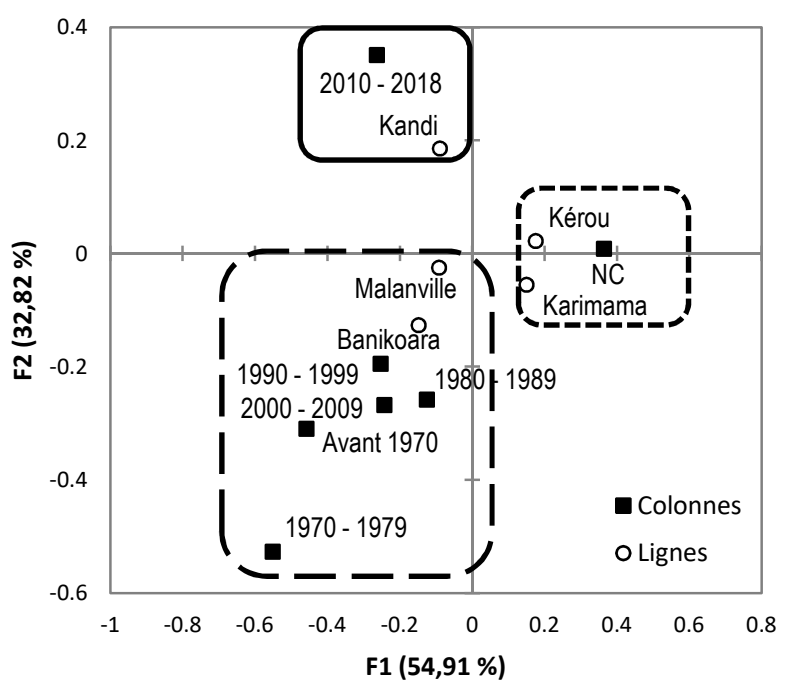

(b) Graphique symétrique (axes F1 et F2: 87,73

Figure 6 : Evolution temporelle de l'identification et de la matérialisation des couloirs

Légende - NC : non connue

L'AFC confirme un lien très significatif entre les communes riveraines et l'évolution dans le temps des initiatives d'identification et de matérialisation des couloirs de transhumance $\left(\mathrm{khi}^{2}=85,06 ; \mathrm{ddl}=24 ; \mathrm{p}\right.$-value $\left.<0,0001\right)$. Cette analyse permet de dégager trois grands regroupements par rapport à la perception des éleveurs transhumants sur l'évolution dans le temps de l'identification et de la matérialisation des couloirs de transhumance (figure 6b). Ces regroupements sont (i) Banikoara et Malanville qui sont pionnières et constantes dans les initiatives d'identification et de matérialisation des couloirs ; (ii) Kérou et Karimama où les transhumants ont peu d'informations sur l'historique de la mise en place des couloirs et; (iii) la commune de Kandi dans laquelle les travaux de sécurisation de couloirs de transhumance sont assez récents.

\section{Mutations spatiales des couloirs de transhumance}

Une dynamique spatio-temporelle est observée au niveau de certains couloirs de transhumance à la périphérie de la RBTW-B ; c'est la perception de $62 \%$ des éleveurs transhumants enquêtés dont $36 \%$ ne donnent aucune explication ; $20 \%$ évoquent l'extension des aires de culture, $4 \%$ l'augmentation des besoins en terre liés à la culture du coton, surtout dans la commune de Banikoara (figure $7 \mathrm{a}$ ). Par contre, 14\% soutiennent que les couloirs sont demeurés statiques ; $12 \%$ ne donnent pas d'explication, $0,9 \%$ justifient la situation par l'extension des aires de culture et $0,8 \%$ par la bonne entente entre agriculteurs et éleveurs.

L'analyse factorielle des correspondances (figure $7 \mathrm{~b}$ ) révèle un lien significatif entre les communes riveraines et les mutations spatiales des couloirs de transhumance $\left(\mathrm{khi}^{2}=49,00 ; \mathrm{ddl}=32 ; \mathrm{p}\right.$-value $\left.<0,0278\right)$. La perception des mutations spatiales des couloirs par les éleveurs transhumants permet de faire trois regroupements. Kandi et Kérou ont des traits communs ; d'une part, des couloirs sont dynamiques et les éleveurs n'en donnent pas la raison ; d'autre part, des couloirs sont statiques à cause de l'extension des aires de culture. Les couloirs sont dynamiques dans Banikoara du fait de l'augmentation des besoins en terre liés à la culture du coton, de la menace des agriculteurs, de l'obstruction des couloirs liée à la recherche de terres fertiles. Enfin, dans Karimama et Malanville, la situation est moins homogène ; des couloirs sont dynamiques à cause de l'extension des aires de culture et l'installation anarchique des agriculteurs ; il y a également des couloirs statiques ; les éleveurs n'en donnent aucune explication ou évoquent la bonne entente entre éleveurs et agriculteurs. 


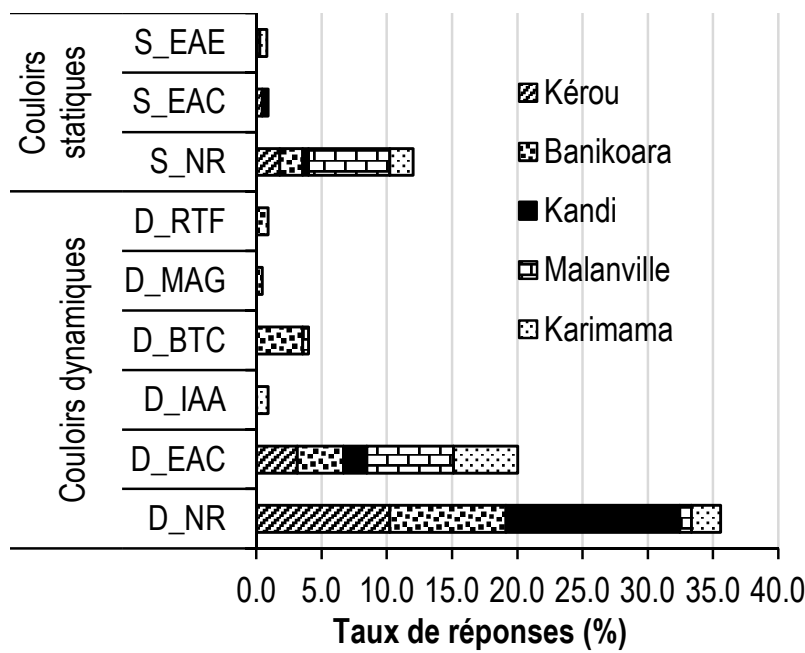

(a) Diagramme en bâtons superposés

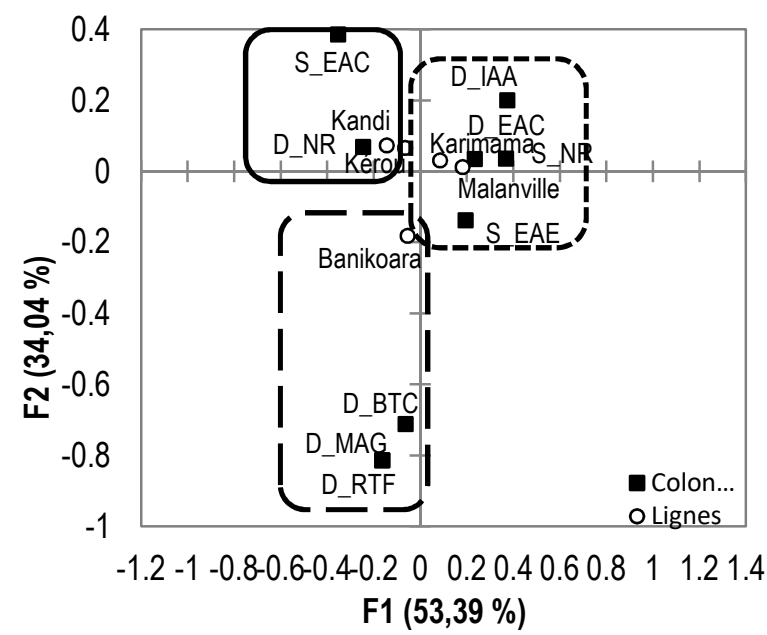

(b) Graphique symétrique (axes F1 et F2 : $87,43 \%$ )

Figure 7 : Mutations spatiales des couloirs de transhumance et causes desdites mutations

Légende : $D$ : couloirs dynamiques ; $S$ : couloirs statiques ; NR : raisons non révélées ; EAC : extension des aires de cultures ; IAA : installation anarchique des agriculteurs ; BTC : augmentation des besoins en terre liés à la culture du coton ; MAG : menace des agriculteurs ; RTF : obstruction des couloirs liée à la recherche de terres fertiles ; EAE : entente entre agriculteurs et éleveurs.

Evolution numérique et/ou actualisation des itinéraires de transhumance : Le nombre d'itinéraires empruntés par les animaux en transhumance à la périphérie de la RBTW-B régresse avec le temps selon $73 \%$ des éleveurs enquêtés. Respectivement 36,29 et $8 \%$ des éleveurs ont fait le constat de cette situation depuis 5 ans (2012), 10 ans (2007) et 15 ans (2002). Les principales explications de la baisse du nombre d'itinéraires de transhumance sont l'extension anarchique des aires de culture (55\% des avis), la culture extensive de coton (9\%), le manque d'aménagement $(5 \%)$, les effets néfastes des feux de végétation sur les ressources fourragères $(3 \%)$ (Figure $8 a)$. L'AFC révèle un lien significatif entre les communes riveraines et les causes de régression du nombre d'itinéraires de transhumance $\left(\mathrm{khi}^{2}=78,10 ; \mathrm{ddl}=28 ; \mathrm{p}\right.$ - value $<0,0001)$. La perception des causes de diminution des possibilités de choix des éleveurs rencontrés permet de distinguer trois groupes (Figure 8b). Ces causes sont

(i) l'extension anarchique des aires de culture et accessoirement, le manque d'aménagements, la non application des sanctions à l'encontre des contrevenants au non-respect des itinéraires de transhumants d'après la perception des transhumants rencontrés dans les communes de Kandi et Malanville ;

(ii) la pression démographique, la culture extensive de coton, les conflits ou la pression des forestiers pour les éleveurs enquêtés dans Banikoara ;

(iii) les causes perçues par transhumants enquêtés dans Kérou et Karimama sont les effets néfastes des feux de végétation ou la mauvaise exploitation des ressources fourragères. 


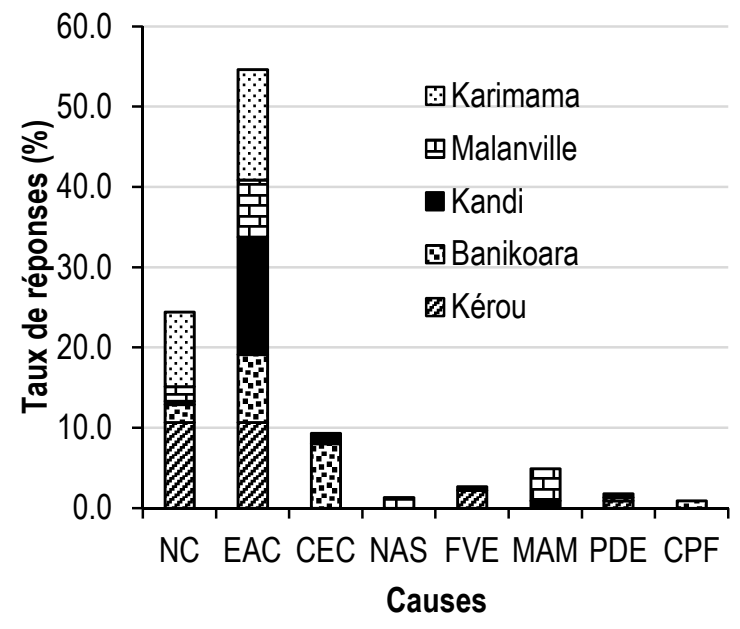

(a) Diagramme en bâtons superposés

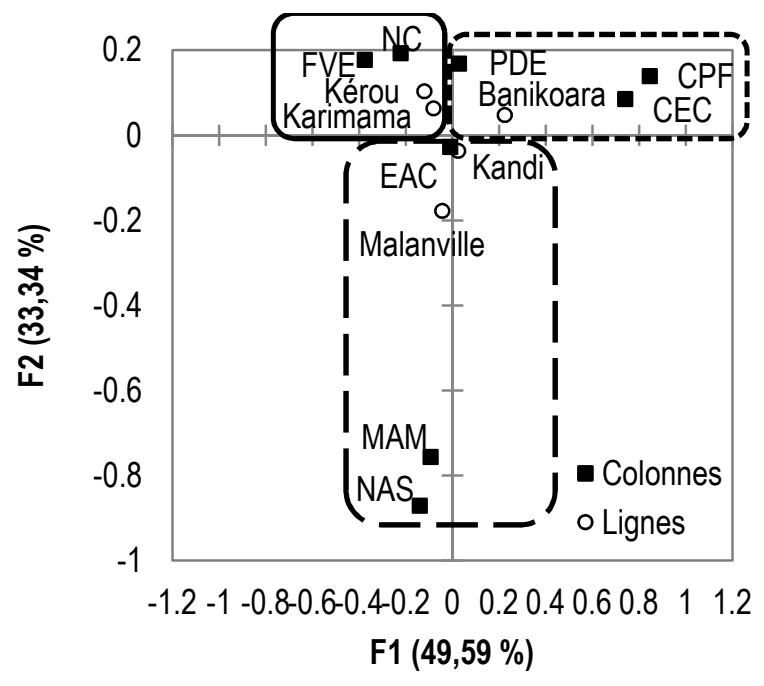

(b) Graphique symétrique (axes F1 et F2 : 82,92

Figure 8 : Causes de réduction du nombre d'itinéraires de transhumance autour de la RBTW-B

Légende : NC : non connue; EAC : extension anarchique des aires de culture; CEC : culture extensive de coton ; NAS : non application des sanctions ; FVE : effets néfastes des feux de végétation ou mauvaise exploitation des ressources fourragères ; MAM : manque d'aménagement ; PDE : pression démographique ; CPF : conflits ou pression des forestiers.

L'actualisation des itinéraires de transhumance ne se fait jamais $(21 \%$ des avis), se fait rarement $(21 \%)$, se fait souvent $(20 \%)$ ou se fait régulièrement $(19 \%)$. Mais, $20 \%$ des éleveurs ne se sont pas prononcés sur cette question. Une analyse factorielle des correspondances (figure 9a) montre un lien très significatif entre la perception des éleveurs des communes riveraines de la RBTW-B et la situation de l'actualisation des itinéraires de transhumance $\left(\mathrm{khi}^{2}=78,15 ; \mathrm{ddl}=16 ; \mathrm{p}\right.$-value < $0,0001)$. Cette analyse indique les itinéraires de transhumance sont régulièrement actualisés dans la commune de Banikoara ; dans les communes de Kandi et Malanville, les itinéraires sont souvent ou rarement actualisés; à Karimama, les actualisations ne sont presque jamais faites. Quelle que soit la fréquence d'actualisation des itinéraires de transhumance, les principales raisons justifiant ces travaux sont la dégradation des aires de pâturage (35\%), l'obstruction des couloirs (32\%), les conflits (19\%) et l'assèchement des points d'eau (15\%). L'AFC (figure 9b) indique un lien significatif entre les justifications des éleveurs et les communes riveraines $\left(\mathrm{khi}^{2}=31,30 ; \mathrm{ddl}=12 ; \mathrm{p}\right.$-value < 0,0018). II s'agit essentiellement de l'obstruction des couloirs pour les transhumants rencontrés dans Banikoara, de la dégradation des aires de pâturage dans Malanville, des conflits pour les éleveurs de Kérou et Karimama et, de l'assèchement des points d'eau pour les transhumants de Kandi. 


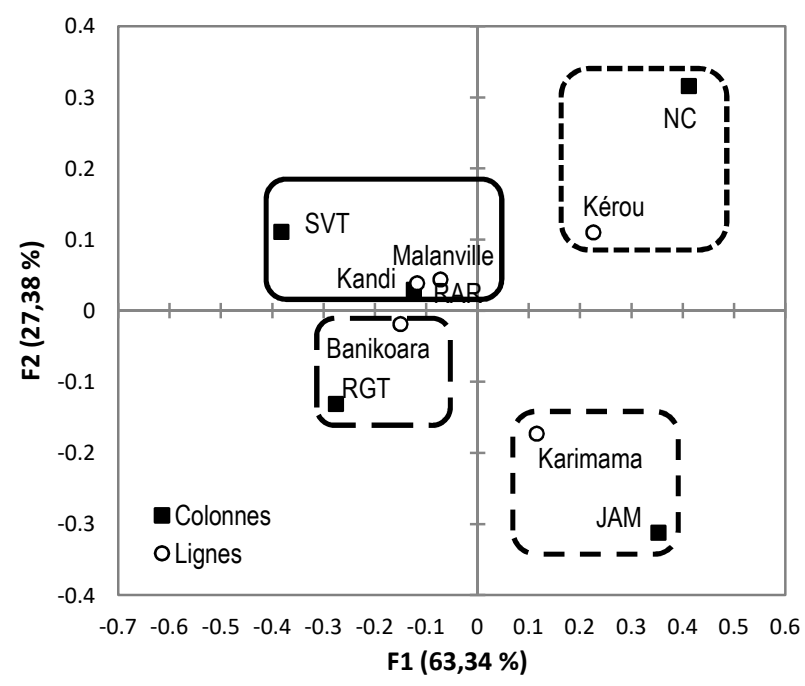

(a) Graphique symétrique fréquence des actualisations (axes F1 et F2 : 90,72\%)

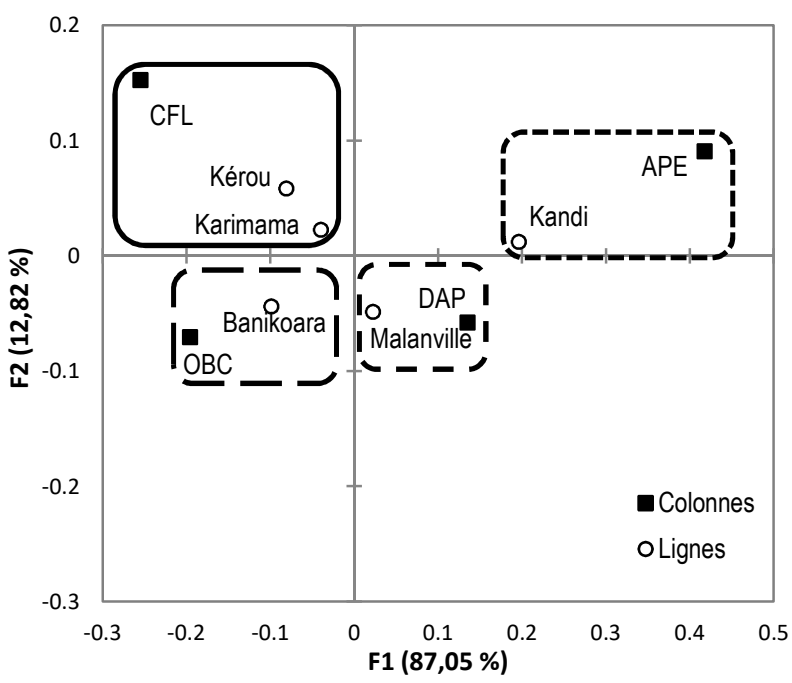

(b) Graphique symétrique des justifiant les actualisations (axes F1 et F2 : 99,87 \%)

Figure 9 : Fréquence d'actualisations des itinéraires de transhumance et justifications

Légende : NC : non connue ; JAM : jamais ; RAR : rarement ; SVT : souvent ; RGT : régulièrement ; DAP : dégradation des aires de pâturage ; OBC : obstruction des couloirs ; CFL : conflits ; APE : assèchement des points d'eau.

\section{DISCUSSION}

Déterminants historiques et bases culturelles de la mise en place des itinéraires de transhumance : La mise en place des itinéraires de transhumance est ancienne; cette pratique d'élevage existait déjà au XIXème siècle et répondait au meilleur usage du milieu naturel (Bani Bio Bigou, 1993). Les éleveurs transhumants, avant la mise en place des réserves de biosphère, suivaient des axes de déplacement traversant celles-ci. Cette activité, perçue par eux comme culturelle, permettait le maintien des liens d'amitié et aussi d'échanges entre les différentes communautés. Ces échanges, perçus comme nécessaires et complémentaires, sont d'ordre transnational (Amadou, 2008). Malgré la pression de surveillance du W, ces couloirs continueraient d'être utilisés à l'intérieur de la réserve (entre le Burkina Faso et la zone de Kaobagou dans la commune de Kérou; entre Karimama et Founougo dans la commune de Banikoara) par certains transhumants indélicats. Les équipes de patrouille de la Direction du parc W au Bénin, conscientes de ce fait, affirment maîtriser la situation de plus en plus (Kpérou Gado, 2006). Pourtant, certains circuits de transhumance intégrant les réserves de biosphère sont récents et tributaires des conditions écologiques des différentes régions (Amadou, 2008). Les épisodes de sécheresse qui ont marqué les dernières décennies ont accentué et parfois tragiquement révélé la gravité du problème écologique auquel doivent faire face de nombreuses sociétés pastorales (Akpo, 2005). Les années 1970 et 1980 sont, en effet, les décennies au cours desquelles les initiatives de matérialisation des couloirs de transhumance ont été remarquables selon les résultats de la présente étude.

Déterminants environnementaux des itinéraires de transhumance : Pour le choix des itinéraires, les éleveurs recherchent des informations relatives à l'existence et à la qualité des pâturages, la disponibilité de l'eau d'abreuvement, l'existence des pistes de transhumance et la présence ou pas de cultures (Diop, 2012). Les travaux de Kpérou Gado et al. (2020) ont permis de révéler que les familles d'essences végétales les mieux représentées sur les itinéraires de transhumance à la périphérie de la RBTW-B sont (i) physiologiquement les Poaceae, les LeguminoseaPapilionoideae, les Combretaceae, les LeguminosaeCaesalpinioideae, les Leguminosae-Mimosoideae, les Rubiaceae; (ii) quantitativement, les Combretaceae, Leguminosae-Caesalpinioideae, les LeguminosaeMimosoideae, les Rubiaceae, les Poaceae, soit, $77,02 \%$ de l'ensemble du cortège. La connaissance de la valeur fourragère des pâturages et de l'offre fourragère liée à l'abondance des Poaceae (riches en énergie), des Rubiaceae et Leguminosae (riches en protéines) permet aux éleveurs de constituer leurs itinéraires (Djenontin, 2010) ; cela justifie le choix de la périphérie de la RBTW$B$ par les transhumants. Les itinéraires de 
transhumance ont tendance à être plus longs et plus dispersés dans les années 1970 en raison de la fréquence accrue des sécheresses et l'expansion des cultures dans les couloirs du bétail. Les éleveurs transhumants sont contraints de créer des itinéraires alternatifs avec une certaine préférence pour ceux qui leur permettent d'atteindre la zone d'accueil le plus rapidement possible et dans de bonnes conditions d'affourragement de leur bétail (Diop, 2012). Un lien significatif est mis en exergue par la présente étude entre itinéraires de transhumance et réseau de points d'eau, lisière des forêts, bordures des champs, voies de communication. Les facteurs biophysiques et climatiques sont déterminants quels que soit les itinéraires de transhumance considérés. Ces résultats corroborent ceux du CORAF/WECARD (2015) et Kpérou Gado (2006) selon lesquels les conditions agroclimatiques et de la disponibilité des ressources pastorales, à savoir pâturages naturels, résidus de culture et eau sont les principales causes citées par les transhumants pour justifier le repositionnement ou l'actualisation des couloirs de transhumance.

Déterminants socio-économiques des itinéraires de transhumance : Pour faciliter le mouvement des animaux en transhumance et garantir le déroulement de cette pratique d'élevage dans des conditions sociales apaisées, l'actualisation des itinéraires s'impose souvent. D'après Diop (2012), les itinéraires et les zones d'accueil sont constituées d'une série d'étapes soigneusement choisies à partir des informations collectées auprès de plusieurs informateurs et de l'expérience personnelle de l'éleveur transhumant acquise des années durant. Une cause sociale majeure est évoquée au cours des présents travaux par les éleveurs pour justifier le repositionnement des couloirs ; il s'agit des conflits. En effet, à l'ancien système de transhumance, à la fois stable et spécifique et basé sur la précision des itinéraires de transhumance, des espaces pastoraux suffisants s'est substitué un système caractérisé par des relations de plus en plus conflictuelles entre agriculteurs et éleveurs et une anthropisation accrue du milieu (Katè et al., 2015; Banoin et Jouve, 2000). Ces conflits résultent des mutations spatiales imposées notamment par l'extension des aires de cultures, l'installation anarchique des agriculteurs, l'augmentation des besoins en terre liés à la culture du coton, la menace des agriculteurs, l'obstruction des couloirs liée à la recherche de terres fertiles. Du coup, il y a une régression des possibilités de choix d'itinéraires par les transhumants d'année en année à la périphérie de la RBTW-B. Les transhumants ont un terroir d'attache ou un « chez eux "; mais à partir du moment où ils se déplacent ils basculent dans une autre catégorie, ils deviennent des éleveurs allochtones, statut qui les rends très vulnérables quand ils ont besoin de se défendre (Schönegg et al., 2006). Conscients de cette perception dans les zones d'accueil, ces derniers valorisent leur savoir, leur savoir-faire et leurs capacités socioprofessionnelles de manière à minimiser les risques encourus (conflits, vols, tracasseries, banditisme). Les services de reconnaissance fournis par les « Ruggas », leaders pour l'organisation et la gestion de la transhumance sont d'une grande utilité en la matière ; ils détiennent des pouvoirs de réglementation et d'imposition de sanctions aux transhumants nonrespectueux des règles et sont soutenus dans cette tâche par des « Garsos » dont le rôle est de coordonner les groupes de transhumants (CORAF/WECARD, 2015). Les éleveurs transhument pour accroître aussi la productivité des troupeaux et les revenus. Les itinéraires de transhumance ont ainsi un lien significatif avec la position des marchés à bétail, des parcs de vaccination, des cabinets et pharmacies vétérinaires. Selon Kpérou Gado (2006), la transhumance est un facteur d'échange de produits d'élevage; elle a des incidences économiques notamment sur la vie des ménages, l'animation des marchés de bétail, la création d'emplois. Place des institutions dans le déterminisme des itinéraires de transhumance : Pour décrire les couloirs de passage, certains transhumants font référence aux structures officielles locales, nationales ou sous régionales. Selon Katè et al. (2014), au niveau local, les mairies, les services techniques du ministère en charge de l'élevage, de l'environnement, les organisations d'éleveurs, les partenaires techniques et financiers appuient la délimitation des couloirs, des zones de pâturage, des points d'abreuvement pour la transhumance. Conformément à l'article 10 de la loi $n^{\circ}$ 2018-20 du 23 avril 2019, l'Etat et les collectivités territoriales prennent des mesures pour réaliser des aménagements concourant à un bon déroulement de la transhumance et à la préservation des réserves naturelles ou aménagées (République du Bénin, 2019). La décision A/DEC.5/10/98 de la Communauté Economique des Etats de l'Afrique de l'Ouest en son article 7, fait obligation que le déplacement des animaux transhumants se fasse par les pistes de transhumance définies par les Etats, conformément à l'itinéraire prescrit sur le certificat international de transhumance (CEDEAO, 1998). L'article $2 \mathrm{du}$ règlement C/REG.3/01/03 relatif à la mise en œuvre de la 
règlementation de la transhumance entre les Etats membres de la CEDEAO stipule que les études d'actualisation des pistes de transhumance et les zones de parcours au niveau sous-régional doivent se réaliser en collaboration avec l'Union Economique et Monétaire Ouest Africaine (UEMOA), le Comité Permanent InterEtats de Lutte contre la Sécheresse dans le Sahel (CILSS) et les autres organisations concernées (CEDEAO, 2003). Dans le souci d'élaborer des mesures urgentes en rapport avec les pistes et les couloirs de transhumance dans la zone périphérique d'influence du Parc Régional du W une réunion ministérielle sur la transhumance transfrontalière s'est tenue à Cotonou le 26 février 2004 entre le Bénin, le Burkina Faso et le Niger. Cette réunion a abouti aux "Accords de Cotonou » qui ont permis la mise en œuvre d'une stratégie d'information, éducation et communication (IEC) au profit des acteurs de la transhumance et l'officialisation de cinq (05) axes de transhumance sous régionaux (Billand et al., 2004). Trois (03) de ces couloirs traversent la zone d'étude ; il s'agit des axes 3, 4 et 5 . Ces accords pourraient justifier l'engouement pour les initiatives d'officialisation des couloirs internes de raccordement aux couloirs sous-régionaux de la

\section{CONCLUSION}

Les déterminants des itinéraires de transhumance à la périphérie de la RBTW-B sont culturels, historiques, socio-économiques, environnementaux et institutionnels. A l'ancien système de transhumance, à la fois stable, basé sur des itinéraires précis, des espaces pastoraux suffisants, des groupes sociaux en parfaite harmonie entre eux, s'est substitué un système caractérisé par une instabilité constante, une anthropisation accrue du milieu, des relations de plus en plus conflictuelles entre agriculteurs et éleveurs. Dans ce contexte, les années à venir, les éleveurs devront faire face à une pression agricole croissante, à des contraintes environnementales plus fortes, aux

\section{REMERCIEMENTS}

Les auteurs témoignent leur gratitude au Laboratoire de Cartographie (Lacarto) de l'Université d'Abomey-Calavi qui a facilité la présente étude dans le cadre d'une thèse

\section{REFERENCES BIBLIOGRAPHIQUES}

Adomou AC, Sinsin B, van der Maesen LJG, 2006. Phytosociological and chorological approaches to phytogeography: a mesoscale study in Benin. Syst. Geogr. Pl., 76 : 155-178. https://www.jstor.org/stable/20649708
CEDEAO à partir de la décennie 2000-2009, tels que présentés par la présente étude. Le cas de la commune de Banikoara où des travaux de géolocalisation des itinéraires de transhumance ont été entrepris dans tous les arrondissements en vue d'améliorer la mobilité du bétail et réduire les conflits entre les acteurs de la transhumance est illustratif (Mairie de Banikoara, 2017). Selon les transhumants enquêtés, cette actualisation ne se fait presque jamais dans la commune de Karimama, se fait souvent ou rarement dans les communes de Kandi et Malanville. Plusieurs auteurs justifient de tels dysfonctionnements au Bénin par, notamment, la cogestion très conflictuelle des infrastructures, la non tenue des assemblées générales des comités de gestion, le manque de formation en gestion des membres des comités, les conflits liés à la gestion financière des revenus de ces infrastructures, l'absence de normes pour orienter la gestion des couloirs de passage (Tama et Mossi, 2011 ; Djenontin et al, 2012). Enfin, dans le choix des itinéraires de transhumance, la présence des forces de défense et de sécurité dans la zone est un critère pris en compte par les éleveurs. Ces derniers évitent de faire face à d'éventuelles tracasseries, notamment celles policières (Diop, 2012).

changements politiques et institutionnels et à l'évolution de la législation foncière. Le pastoralisme pourrait alors évoluer vers l'agro-pastoralisme, la sédentarisation ou se maintenir dans un système où la mobilité sera principalement au cœur des stratégies, à condition qu'elle soit mieux accompagnée. En termes de perspectives de recherches, il va s'en dire qu'il faudra approfondir les investigations sur les forces et faiblesses des changements envisageables, les appuis pouvant être apportés aux décideurs notamment avec l'utilisation des SIG, des outils cartographiques et des enquêtes socioéconomiques.

de doctorat. Ils adressent également leurs remerciements aux reviewers pour les contributions pertinentes dans l'amélioration du document.

Akpo LE, 2005. Ecologie pastorale. UCAD-FST, Dakar. $58 \mathrm{p}$.

Amadou $B, 2008$. Réserves de biosphère en Afrique de l'Ouest: vers des modèles de développement 
durable. UNESCO-MAB/PNUE-FEM - Nouvelle Imprimerie du Niger (NIN), Niamey. $62 \mathrm{p}$.

Banoin $M$ et Jouve $P, 2000$. Déterminants des pratiques de transhumance en zone agro-pastorale sahélienne: cas de l'arrondissement de Mayahi, au Niger. Options Méditerranéennes, Sér. A / $n^{\circ} 39$, Rupture... nouvelle image de l'élevage sur parcours.

Bani Bio Bigou L, 1993. Elevage transhumant et mobilité transfrontière dans la vallée du Niger, Cas de la frontière Bénin-Niger. Unité de recherches associées UNB-ORSTOM, Cotonou. $25 \mathrm{p}$.

Billand A, De Visscher MN, Kidjo FC, Compaore A, Boureima A, Morel A, Camara L, 2004. Mission d'appui au montage du « Plan d'Aménagement et de Gestion de la Réserve de Biosphère Transfrontalière W - 2006-2010 ». Volume I : Etat des lieux. Rapport provisoire, Coordination régionale ECOPAS, Ouagadougou. $211 \mathrm{p}$.

CEDEAO, 2003. Règlement C/REG.3/01/03 relative à la mise en œuvre de la réglementation de la transhumance entre les Etats membres de la Communauté Economique des Etats de l'Afrique de l'Ouest. Dakar. $5 \mathrm{p}$.

CEDEAO, 1998. Décision A/DEC.5/10/98 relative à la réglementation de la transhumance entre les Etats membres de la Communauté Economique des Etats de l'Afrique de l'Ouest. Abuja. 7 p.

Codjia V, 2016. Revue des filières bétail/viande \& lait et des politiques qui les influencent au Bénin. Rapport de consultation, Organisation des Nations Unies pour I'Alimentation et l'Agriculture (FAO) et Communauté Economique des Etats de l'Afrique de l'Ouest (CEDEAO). $47 \mathrm{p}$.

CORAF/WECARD, 2015. Transhumance transfrontalière et conflits liés à l'utilisation des ressources naturelles en Afrique de l'Ouest. CORAF/WECARD, Dakar. $94 \mathrm{p}$.

Dagnelie $P, 2007$. Statistique théorique et appliquée Tome I. Statistique descriptive et bases de l'inférence statistique. Bruxelles, Editions De Boeck. $511 \mathrm{p}$.

DPA, 2016. Annuaire statistiques de la Direction de la Production Animale. Cotonou. 121 p.

Diop AT, 2012. La transhumance transfrontalière en Afrique de l'Ouest: Proposition de plan d'action. Rapport de Consultation, Organisation des Nations Unies pour I'Alimentation et l'Agriculture (FAO). $139 \mathrm{p}$.
Djènontin AJ, Baco NM, Akponikpe IP, 2012. Evaluation des infrastructures pastorales et agropastorales du département de l'Alibori, au Nord-Est du Bénin. Rapport de consultation, APIDev-ONG, Parakou. $65 \mathrm{p}$.

Djènontin $A J, 2010$. Dynamique des stratégies et pratiques d'utilisation des parcours naturels pour l'alimentation des troupeaux bovins au Nord-Est du Bénin. Thèse de Doctorat, Université d'Abomey-Calavi. 203 p.

Djènontin AJ, Houinato $M$, Toutain $B$, Sinsin $B, 2009$. Pratiques et stratégies des éleveurs face à la réduction de l'offre fourragère au Nord-Est du Bénin. Sécheresse, 20 (4) : 346-53.

Duteurtre G. et Faye B, 2009. L'élevage, richesse des pauvres : stratégies d'éleveurs et organisations sociales face aux risques dans les pays du Sud. Editions Quae, Versailles. $284 \mathrm{p}$.

Eboh EC, Oji KO, Oji OG, Amakom U, Ujah OC, 2008. Towards the ECOWAS Common Agricultural Policy Framework: Nigeria Case Study and Regional Analysis. African Institute for Applied Economics: Enugu, Nigeria. 197 p.

Houessou LG, Teka O, Toko Imorou I, Lykke AM, Sinsin $B, 2013$. Land Use and Land-Cover Change at "W" Biosphere Reserve and Its Surroundings Areas in Benin Republic (West Africa). Environment and Natural Resources Research, 3 (2): $87-101$. DOI: http://dx.doi.org/10.5539/enrr.v3n2p87

Kagoné $H$, Toutain $B$, Dulieu $D$, Houinato $M$, Boureima A, Nocker U, 2006. Pastoralisme et Aires protégées en Afrique de l'Ouest: Du conflit à la gestion concertée de la transhumance transfrontalière dans la région du parc $W$ (Bénin, Burkina-Faso, Niger). Bull Animal. Hlth. Production. Afr, 54 : 43-52.

Katé S, Dagbenonbakin GD, Agbangba CE, de Souza JF, Kpagbin G, Azontondé A, Ogouwalé E, Tente B, Sinsin B, 2014. Perceptions locales de la manifestation des changements climatiques et mesures d'adaptation dans la gestion de la fertilité des sols dans la Commune de Banikoara au Nord-Bénin. Journal of Applied Biosciences, 82 : 7418-7435.

Katè $S$, Hounmènou GC, Amagnide $A$, Houndonougbo PV, Tchobo A, Tente B, Diarra S, Sinsin B, 2015. Changement climatique, mécanisme actuel de prévention et de gestion des conflits entre agriculteurs et éleveurs en zone agro pastorale de production cotonnière au nord 
Bénin : cas de la Commune de Banikoara. African Crop Science Journal, 23 (1) : 9-26.

Kpérou Gado BO, Toko Imorou I, Arouna O, Oumorou M, 2020. Caractérisation des parcours de transhumance à la périphérie de la réserve de biosphère transfrontalière du $\mathrm{W}$ au Bénin. Int. J. Biol. Chem. Sci. 14 (2) : 333-352. DOI: https://dx.doi.org/10.4314/ijbcs.v14i2.3

Kpérou Gado BO, 2006. Impacts socio-économiques de la transhumance transfrontalière dans la zone riveraine du Parc W du Bénin. Thèse Méd. Vét., EISMV-Dakar. $114 \mathrm{p}$.

Lesse P, Houinato M.B, Djenontin J, Dossa H, Yabi B, Toko I, Tente B, Sinsin B, 2015. Transhumance en République du Bénin : états des lieux et contraintes. Int. J. Biol. Chem. Sci. 9(5): 26682681.

DOI http://dx.doi.org/10.4314/ijbcs.v9i5.37

Mairie de Banikoara, 2017. Fiche d'identification des infrastructures agropastorales: couloirs de passage, aires de pâturage, points d'abreuvement, centres de vaccination. Rapport provisoire d'étude, Mairie de Banikoara. $206 \mathrm{p}$.

Météo Bénin, 2018. Données climatiques des stations météorologiques de Banikoara, Kérou, Kandi, Karimama, Malanville, Natitingou. Agence Nationale de Météorologie, Cotonou.

Nori M, Taylor M, Sensi A, 2008. Droits pastoraux, modes de vie et adaptation au changement climatique.Londres : Institut International pour l'Environnement et le Développement (IIED), dossier $n^{\circ} 148.28 p$.

République du Bénin, 2019. Loi n²018-20 du 23 avril 2019 portant code pastoral en République du Bénin. Cotonou. $23 p$.

Schönegg G, Martel P, Sano B, Noufou S, 2006. Les conflits liés à la transhumance transfrontalière entre le Niger, le Burkina Faso et le Bénin. Rapport d'expertise, Service Allemand de Développement (DED), Division Développement Rural, Gestion des Eaux et des Ressources Naturelles. $73 p$.

Tama C. et Mossi A, 2011. Rapport de l'état des lieux des infrastructures et aménagements agropastoraux dans quelques communes du département du Borgou. Rapport de consultation, APIDev-ONG, Parakou. 186 p.

Tente BHA, Toko Imorou I, Lougbegnon T, Boni Y, 2012. Réalisation de l'étude de référence sur le potentiel des parcours naturels et des infrastructures d'accueil des troupeaux transhumants au Bénin. Rapport de consultation, Direction Générale des Affaires Intérieures (DGAI). $31 \mathrm{p}$.

Zakari S, Tente BAH, Yabi I, Toko Imorou I, Tabou T, Afouda F, N'Bessa B, 2015. Vulnérabilité des troupeaux transhumants aux mutations climatiques : analyse des perceptions et adaptations locales dans le bassin de la Sota à Malanville. Afrique Science 11 (3) : 211-228. 